\title{
REPAIR OF ADULT INGUINAL HERNIA WITH CONTINUOUS SUTURES OF EXTERNAL OBLIQUE APONEUROSIS
}

\author{
Sunilkumar Singh Salam¹, N. Jitendra ${ }^{2}$, Rosemary Vumkhoching ${ }^{3}$, Renuca Karam ${ }^{4}$, T. Arun Kumar Singh ${ }^{5}$ \\ ${ }^{1}$ Assistant Professor, Department of Surgery, Regional Institute of Medical Sciences, Imphal, Manipur. \\ 2 Professor, Department of Surgery, Jawaharlal Nehru Institute of Medical Sciences, Imphal, Manipur. \\ ${ }_{3}^{3}$ Associate Professor, Department of Surgery, Jawaharlal Nehru Institute of Medical Sciences, Imphal, Manipur. \\ ${ }_{4}^{4}$ Assistant Professor, Department of Anatomy, Jawaharlal Nehru Institute of Medical Sciences, Imphal, Manipur. \\ 5 Professor, Department of Surgery, Jawaharlal Nehru Institute of Medical Sciences, Imphal, Manipur.
}

\section{ABSTRACT}

\section{OBJECTIVE}

To study the repair of adult inguinal hernia with continuous sutures of external oblique aponeurosis with respect to its acceptability, effectiveness, outcome and the satisfaction of patients.

\section{METHODOLOGY}

The observational study was conducted from August 2008 to September 2010 in Surgery Unit IIA of the Department of General Surgery, Regional Institute of Medical Sciences, Hospital, Imphal. Adult patients with inguinal hernia (18-75 years) were admitted and treated with continuous sutures of external oblique aponeurosis as originally described by Desarda in 2001.

\section{RESULTS}

There were 54 patients - 51 males (94.44\%) and 3 females (5.6\%). Age of the patient's ranges from 18-75 years (Mean age of 53.9 years). Out of the 54 patients, 32 patients (59.3\%) were having indirect inguinal hernia, 19 patients (35.2\%) with direct inguinal hernia and 3 patients (5.6\%) had combined direct and indirect inguinal hernias. Right sided hernia was seen in 37 patients $(68.5 \%)$ and left sided hernia in 17 patients (31.5\%); 47 patients (87.03\%) were operated under Spinal Anaesthesia (SA) and 7 patients (12.96\%) under epidural anaesthesia. Adhesions were found in 5 cases $(9.26 \%)$, four patients (7.41\%) had transient wound oedema, 3 patients $(5.6 \%)$ had pus collection followed by drainage, 3 (5.6\%) patients had wound haematoma, which subsided by itself and $2(3.70 \%)$ had seroma formation which required regular dressing. During the follow-up period, no hernia recurrence was encountered. Majority of the patients had mild-to-moderate post-operative pain, which was easily managed with analgesics. The hospital stay was short, thus allowing the patients a quicker return to activity. Majority of the patients (40, i.e. $74.07 \%$ ) were discharged on the 3rd post-operative day. Majority of the patients were satisfied with the operation.

\section{CONCLUSION}

Repair of adult inguinal hernia with continuous sutures of external oblique aponeurosis is safe and effective in terms of postoperative pain, return to work, recurrence, cost effectiveness and can be the preferred choice for open repair of adult inguinal hernias.

\section{KEYWORDS}

Inguinal hernia, Desarda's Technique, External Oblique Aponeurosis.

HOW TO CITE THIS ARTICLE: Salam SS, Jitendra N, Vumkhoching R, et al. Repair of adult inguinal hernia with continuous sutures of external oblique aponeurosis. J. Evolution Med. Dent. Sci. 2016;5(30):1590-1595, DOI: 10.14260/jemds/2016/374

\section{INTRODUCTION}

"Hernia" meaning a rupture in Latin, is defined as a protrusion of a viscus or a part of it through an opening in the wall of the cavity that contains it.

The incidence of abdominal wall hernias are: inguinal (75\%), femoral (8.5\%), umbilical $(15 \%)$ and $1.5 \%$ for the rarer forms. ${ }^{1}$ Inguinal hernias in the adult are acquired defects in the abdominal wall, the common denominator being a weakness in the abdominal wall musculature due to

Financial or Other, Competing Interest: None.

Submission 09-03-2016, Peer Review 22-03-2016,

Acceptance 24-03-2016, Published 14-04-2016.

Corresponding Author:

Dr. Sunilkumar Singh Salam,

Assistant Professor,

Department of Surgery,

Regional Institute of Medical Sciences,

Imphal, Manipur-795004.

E-mail: shoenil24@gmail.com

DOI: $10.14260 /$ jemds $/ 2016 / 374$ chronic coughing, chronic obstructive pulmonary disease, obesity, straining due to constipation or prostatism, pregnancy, birth weight $<1500 \mathrm{gm}$, family history of hernia, defective collagen synthesis, congenital connective tissue disorders, previous right lower quadrant incision, cigarette smoking, heavy weight lifting, etc. Congenital hernias can be considered an impedance of normal development rather than an acquired weakness. ${ }^{2}$

The condensed transversalis fascia and aponeurotic extensions both give mechanical strength to the posterior inguinal wall to resist internal abdominal blows and prevent hernia formation. The strength of the posterior inguinal wall is directly related to the number of aponeurotic fibers it contains and not to the strength of the transversalis fascia alone. Muscular contraction of the transversus abdominis pulls this posterior wall and the aponeurotic extensions upward and laterally creating tension in it to prevent hernia formation. ${ }^{3}$

Inguinal hernia operations are generally based on anatomical considerations keeping aside the physiological 
aspects during the operative procedure. In an attempt to make hernia repair tension free, the use of mesh has been widely advocated. Many patients with inguinal hernia are cured as a result of the current surgical techniques, though factors that prevent hernia formations are not restored. Of late chronic groin pain and the surge of mesh migration and its associated ailments have been a major concern apart from the high cost factor. Hence, the surgical physiology of the inguinal canal needs to be reconsidered.

A physiologically dynamic and strong posterior inguinal wall and the shielding and compression action of the muscles and aponeuroses around the inguinal canal are important factors that prevent hernia formation or hernia recurrence after repair. In addition, the squeezing and plugging action of the cremaster muscle and binding effect of the strong cremasteric fascia also play an important role in the prevention of hernia.

In a developing economy where the cost of operation and loss of working days in the service sector due to postoperative morbidity is a primary concern, the non-mesh adult inguinal hernia repair with continuous sutures of external oblique aponeurosis promises to get rid of these impediments to quality health care by reducing the operating time for the large number of patients, decreased immediate and long-term post-operative morbidity, earlier return to full time employment and thus reduce the net cost of the operation. Laparoscopic repair fails to remedy on the above mentioned factors; being costlier, equipment and expertise dependent, and not easily accessible in settings such as ours. This observational study has been designed to assess the efficacy and the other benefits of adult inguinal hernia repair with continuous sutures of external oblique aponeurosis.

\section{MATERIALS AND METHODS}

The study was conducted in Surgery Unit IIA of the Department of General Surgery, Regional Institute of Medical Sciences (RIMS) Hospital, Imphal, from August 2008 to September 2010. It was an observational study and approved by the Institutional Ethical Committee. Patients between 18 to 75 years of age with primary inguinal hernia with American Society of Anaesthesiologists (ASA).4 Grade I or II on preoperative assessment were included in the study.

Patients having associated medical conditions like uncontrolled diabetes mellitus, uncontrolled hypertension or those patients who were operated on emergency basis and patients not falling in the above mentioned inclusion criteria were excluded.

Under spinal or epidural anaesthesia, an oblique inguinal skin incision, about $6-8 \mathrm{~cm}$ in length, is made $1.25 \mathrm{~cm}$ above and parallel to the medial two-thirds of the inguinal ligament. The External Oblique Aponeurosis (EOA) is then incised along the line of its fibres in line with the upper crus of the superficial inguinal ring [Fig. 1]. The cord structures are then separated from the inferior flap of EOA by blunt dissection and elevated from the inguinal ring en masse with the fingers of one hand at the pubic tubercle so that the index finger can be passed underneath to meet the index finger of the other. In indirect hernias, the hernial sac is identified, dissected up to the internal ring and opened to allow examination of its contents. The contents, if present, are reduced and the sac is transfixed and excised. In direct hernias, the sac is inverted.
The upper leaf of the EOA is then sutured with the inguinal ligament from the pubic tubercle to the deep inguinal ring using Vicryl 1-0/Prolene 1-0 continuous sutures [Fig. 2]. The first two sutures are taken through the anterior rectus sheath, where EOA is fused with it and each suture is passed first through the inguinal ligament, then the transversalis fascia and then the EOA. The last suture is taken so as to narrow the deep inguinal ring sufficiently without constricting the spermatic cord.

A splitting incision is then made in this sutured medial leaf of the EOA, partially separating a strip $1.5-2 \mathrm{~cm}$ wide [Fig. 3]. This splitting incision is extended medially up to the pubic symphysis and $1-2 \mathrm{~cm}$ beyond the deep inguinal ring laterally, keeping the medial insertion and lateral continuation of this strip intact. Then the upper free border of the strip was sutured to the internal oblique muscle/conjoined muscle with Vicryl 1-0/Prolene 1-0 continuous suture all along its length resulting in the strip of the EOA being placed behind the cord to form a new posterior wall of the inguinal canal [Fig. 4]. The spermatic cord was then placed in front of the newly formed posterior wall composed of the strip of EOA and the internal oblique/conjoined muscle. The lateral leaf of the EOA is then sutured to the newly formed medial leaf of the EOA in front of the cord. This was followed by routine closure of the superficial fascia with absorbable sutures and the skin with Vicryl Rapide 3-0 using subcuticular stitches or with silk or skin stapler.

Intraoperative records of duration of operation, amount of blood loss, presence of enterocele, omentocele, adhesions, fibrosis or empty sac and any other incidental intraoperative findings were noted. Pain in the immediate post-operative period was recorded using the Numeric Scale to describe pain and affect on ADLs. 5 (Activities of Daily Living) and after six hours following which the effect of spinal anaesthesia is expected to have weaned at 24 hours and at 48 hours. The patients were motivated to an early ambulation and discharged from the hospital in the absence of surgical site infection, wound seroma and haematoma.

All the patients were operated by consultants. The duration of operation averaged 38.6 minutes (range 35-45 minutes) from skin incision till closure. Those patients taking 45 minutes were because the procedure was being done for the first time in the early stages of the study period and there was a detailed explanation of the procedure to the students.

All the patients were advised to come for follow-up at 2 weeks, 1 month, 6 months and after 2 years following surgery.

\section{RESULTS}

A total of 54 adult patients with inguinal hernia, 51 males and 3 females were included in the study. Mean age was 53.9 years and maximum were between $48-58$ years; 37 patients (68.5\%) had right-sided inguinal hernia, 17 patients $(31.5 \%)$ had left-sided inguinal hernia. There were 32 patients (59.2\%) with indirect inguinal hernias, 19 patients (35.2\%) with direct inguinal hernias, while combined hernia was seen in $3(5.5 \%)$ patients [Table 1$]$.

The commonest presenting symptom was painless reducible swelling in the groin seen in 36 patients $(66.7 \%)$, followed by pain with reducible swelling in the groin seen in 7 cases $(12.96 \%)$. Six patients $(11.1 \%)$ complained of an annoying heavy feeling or dragging sensation and 5 patients $(9.25 \%)$ gave history of strain prior to surgery. Strain was due 
to chronic cough and constipation in 2 patients and lifting of heavy weight in 3 cases. Out of the 36 patients with painless reducible swelling, 4 patients had associated conditions like well-controlled hypertension and 3 had uncomplicated diabetes mellitus.

Spinal anaesthesia was used in 47 patients and epidural anaesthesia was used in 7 patients. The duration of operation of average 38.6 minutes (Range 35-45 minutes) from skin incision till closure. Those patients taking 45 minutes were because the procedure was being done for the first time in the early stages of the study period and there was a detailed explanation of the procedure to the students. There was no excessive bleeding in any of the case [Table 2].

The post-operative pain score ranged from 3 (mild pain) to 7 (moderate pain) at 24 hours after surgery. Majority of the patients, numbering 36 (66.7\%) experienced a pain score of 3 (mild pain), while $16(29.6 \%)$ patients experienced a pain score of 5 (moderate pain) and 2 patients $(3.7 \%)$ had a pain score of 7 (moderate-to-severe pain). Injectable analgesic was used for controlling the pain. At 48 hours, 44 patients $(81.5 \%)$ experienced no pain (Pain score-0), 8 patients $(14.8 \%)$ had mild pain (Pain score-3) and 2 patients $(3.7 \%)$ had moderate pain (Pain score-5). Oral analgesics were given to control the pain [Table 3].

Majority of the patients - 36 (66.7\%) were ambulatory by the 1st post-operative day. Because of the mild pain, they were able to carry out their Activities of Daily Living (ADLs) easily and without any discomfort. The hospital stay following surgery ranged from 2-8 days (mean 4.67 days). Majority of them $(40-74.07 \%)$ were fit for discharge by the 3rd postoperative day. Some of the patients were discharged on the 8th post-operative day after removal of stitches. Because of transportation problem and being from a remote area, 4 patients (7.4\%) wanted to go home after complete removal of stitches and 2 patients $(3.7 \%)$ went home after the culture sensitivity report with appropriate antibiotics.

Four patients $(7.4 \%)$ had transient wound oedema that subsided on its own. Three patients (5.6\%) had superficial wound infections that needed treatment with drainage of pus and antibiotics. Haematoma was observed in 3 patients (5.6\%) who required no treatment. Seroma requiring change of dressing was encountered in 2 patients (3.7\%). There were no patients with chronic groin pain and recurrence in the follow-up of the study period for 2 years. No patients had ischemic orchitis or associated injuries to adjacent organs like bladder and bowel.

The time taken for patients to return to work was 10-14 days (mean 12.76 days). The majority of the patients (93.5\%) were satisfied with the operation. Remaining $6.5 \%$ were not satisfied with the operation due to development of haematoma and wound infection requiring pus drainage.

\section{DISCUSSION}

Inguinal structures, as living entities, cannot be appreciated by either dissection on cadavers or operations on patients under spinal or general anaesthesia. Also imaging of the inguinal canal in patients with or without hernia by ultrasonography is not satisfactory due to the small size of the canal and the fact that all structures are seen black and white.

Demonstration of movements of the posterior wall and the musculo-aponeurotic structures around the inguinal canal during bouts of raised intra-abdominal pressure has not been successful, though an attempt to do so has been done by Tobin et al. 6 on one patient.

The present study shows that adult inguinal hernia is more common once their active life is over, which may be due to the degenerative process in the elderly leading to attenuation and weakening of the connective tissue within the aponeurotic layer of the groin leading to hernia. These results are comparable to the studies conducted by various authors, viz. Desarda MP.7 Situma SM et al. ${ }^{8}$

Rodriguez PL et al. ${ }^{9}$ reported in 2004 on their experience with the hernia repair technique in which the posterior wall of the inguinal canal has been strengthened with a fixed band of the external oblique aponeurosis to produce a physiologically active posterior wall on 56 patients reported $1.7 \%$ incidence of haematocele and late granuloma.

Lichtenstein IL et al.10 1989 in their preliminary report on 1000 consecutive patients with tension free mesh repair reported no incidence of wound infection and two haematoma, which resolved spontaneously.

Desarda MP. ${ }^{11}$ in his study of 229 hernias reported that $94.3 \%$ of the patients reported mild pain for a period of two days and attained free ambulation within a period of 18-24 hours (mean 19.26 hours) with the same technique of hernia repair.

Mitura K et al.12 reported that in the hernia repair using the external oblique aponeurosis, there is a decrease in the duration of operation (56.6:66.55 mins.); less intense postoperative pain (3.3:3.8 on the VAS/Visual Analogue Scale), and faster discharge from the hospital (4:5 days) compared to Lichtenstein tension free hernia repair method.

McCormack K et al. ${ }^{13}$ concluded from his research that the use of mesh during repair of hernia took longer time, appeared to be at a higher risk of serious complication rate in respect of visceral (especially bladder) and vascular injuries, costlier and not easily learnt by trainee surgeons.

Bhattacharjee PK. ${ }^{14}$ in his article describing the techniques of inguinal hernia repair using a strip of EOA with the following results: average hospital stay of 2-3 days, return to work within 1-2 weeks and recurrence of only one out of the 400 patients followed for 10 years. These results are comparable with operations performed with mesh.

Porrero et al. 15 conducted a recent prospective study on Shouldice repair on 775 patients. The average duration of surgery was 57.5 (40-75) minutes. The most significant postoperative complications were urinary retention (8\%), headache $(7 \%)$ and ecchymosis (6\%). While $20 \%$ of the interventions were on an outdoor basis, $76 \%$ of the patients were discharged within 1 day. Average absence from work was 20 days; recurrence rate at 7 years was $2 \%$.

Tanphiphat C. 16 et al. in his prospective study of 120 patients reported that there was no additional benefit regarding post-operative hospital stay and return to full time employment with laparoscopic hernia repair, but had a higher cost.

Earl Shouldice. ${ }^{17}$ in 1953 described a modification to the Bassini's repair, relying on a 4-layer closure through a special continuous suturing technique, thus doubling the fascia transversalis. His series of 8317 hernias over a 10 -year period showed a recurrence rate of $0.8 \%$.

A recent randomized trial with Shouldice repair by Fleming et al.18 suggested a median operation time of 56 minutes, only $48 \%$ of the patients discharged from the 
hospital within 24 hours, rate of complications was $36 \%$, median time taken to return to normal activities was 5 weeks and recurrence at one year was $4.3 \%$.

Neumayer M. ${ }^{19}$ et al. reported that the rate of recurrence of primary inguinal hernia repair by laparoscopic technique has a higher rate of recurrence than that of the open technique in a study of 1983 patients with a followup of more than 2 years.

Nixon SJ. ${ }^{20}$ et al. studied 1361 patients who underwent 1473 inguinal hernia repairs by open mesh (573), open sutured (499) and totally extra peritoneal repair (289) with more than ten years followup. They reported that for open surgery, the best results were obtained after open sutured repair not the open mesh, while both showed no statistically significant difference in the rate of recurrence ( $p>0.9)$.

KuruKahvecioglu 0 et al.21 reported the first case polytetrafluoroethylene (PTFE) mesh migration into the bladder after a bilateral laparoscopic intraperitoneal onlay mesh hernia repair. The patient underwent cystoscopy and laparotomy for excision of the migrated PTFE prosthesis into the bladder.

Goswami $\mathrm{R}$ et al.22 in his study concluded that laparoscopic repair of the hernia was associated with higher incidence of visceral and vascular injury. Particular mention had been made of a case, wherein the Prolene mesh migrated and eroded the caecum

Nicholson S. ${ }^{23}$ observed an increase in the incidence of chronic groin pain from $1 \%$ to $28.7 \%$ and the recurrence rate to increase to more than $2 \%$ on hernia repair. He thus advocated the use of an operation that was simple in technique, easily learned by trainee surgeons, did not require the implantation of a foreign body like mesh and had a recurrence rate of $1-2 \%$ or less.

Hay JM.24 in a multicentre control trial of 1578 patients comparing the various technique of anatomical repair reported the following rates of haematoma: Bassini's 5\% (21/420), McVay 8.35\% (34/407), Shouldice 5.7\% (23/401). Abscess incidence rates were: Bassini's 1.4\% (6/420), McVay 0.7 (3/407), Shouldice 1.2\% (5/401).

Kusnierczyk R et al. ${ }^{25}$ reported on 500 patients with increased recurrence risk group who were subjected to inguinal hernia repair by inguinal canal reconstruction with a fascial flap collected from the medial branch of the aponeurosis of the external oblique abdominal muscle, which strengthens the posterior wall of the inguinal canal preperitoneally. It resulted in good early ambulation without the use of special operating room or equipment, thus reducing the overall cost of treatment.

Desarda MP. ${ }^{26}$ reported a no mesh repair comparable with the mesh repair. The operation was simple, did not require extensive dissection of the inguinal floor as in Bassini or Shouldice or mesh repair and produced excellent results. He thus hypothesised his method as an alternative to mesh or laparoscopic repair in line with Nicholson.

Khan $\mathrm{N}$ et al. ${ }^{27}$ in 2008 reported a post-operative pain score of mild (47.3\%), moderate (37.5\%) and severe (15.2\%) in 112 patients operated with open mesh hernia repair.

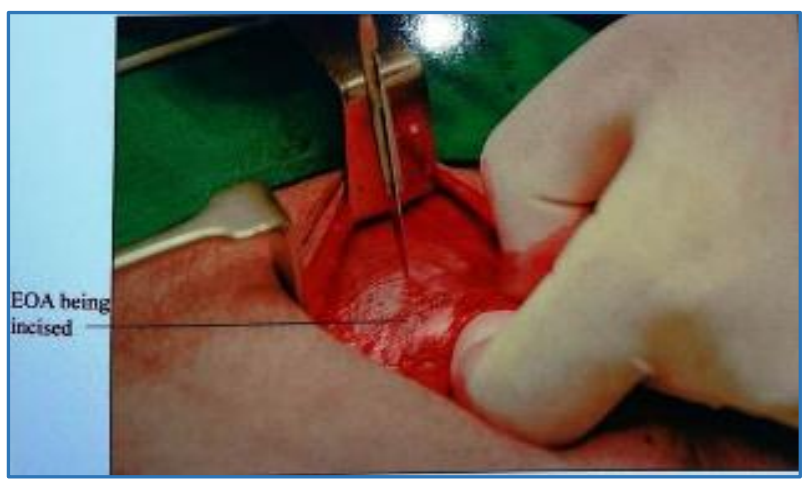

Fig. 1: Photograph showing External Oblique Aponeurosis (EOA) being Incised

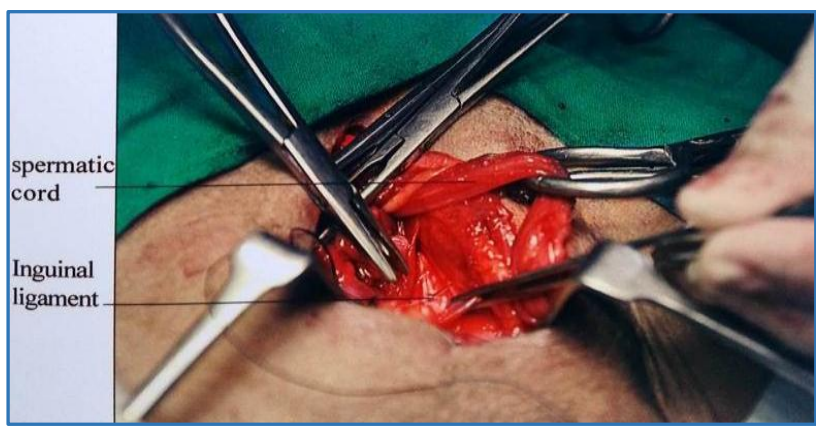

Fig. 2: Photograph showing Upper Leaf of External Oblique Aponeurosis being Sutured to Inguinal Ligament

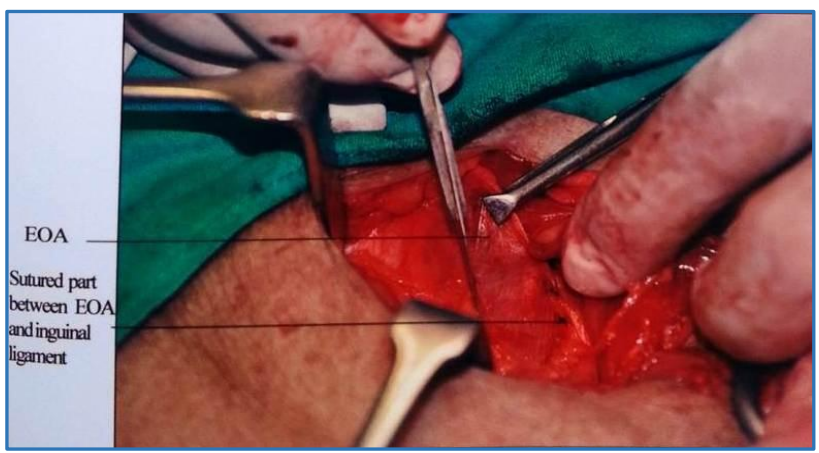

Fig. 3: Photograph showing a Splitting Incision being made in the Sutured Medial Leaf of External Oblique Aponeurosis (EOA) being Incised

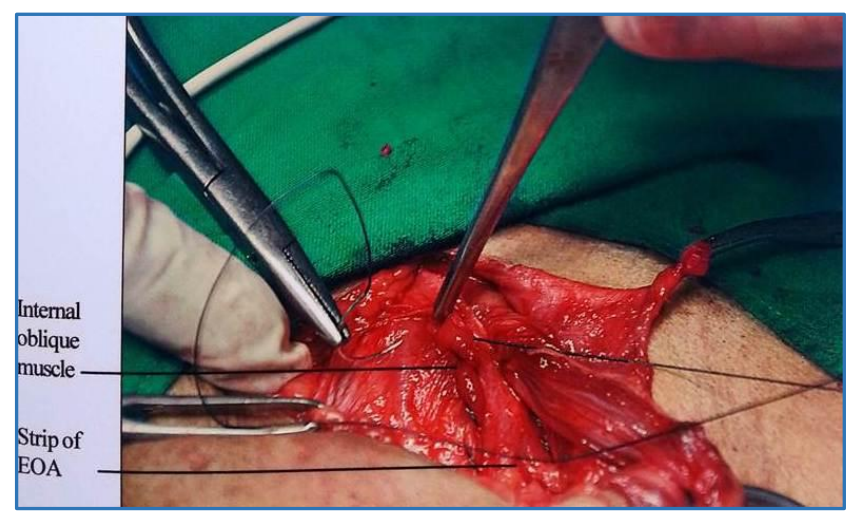

Fig. 4: Photograph showing Upper Free Border of the Strip of External Oblique Aponeurosis (EOA) being Sutured to Internal Oblique Muscle behind the Spermatic Cord to form a new Posterior Wall 


\begin{tabular}{|c|c|c|}
\hline & Types & No. (\%) \\
\hline 1 & Indirect inguinal hernia & $32(59.3 \%)$ \\
\hline 2 & Direct inguinal hernia & $19(35.2 \%)$ \\
\hline 3 & $\begin{array}{c}\text { Combined inguinal hernia } \\
\text { (Indirect + Direct) }\end{array}$ & $3(5.5 \%)$ \\
\hline \multicolumn{2}{|c|}{ Total } & $\mathbf{5 4}(\mathbf{1 0 0 \% )}$ \\
\hline \multicolumn{2}{|c|}{ Table 1: Types of Inguinal Hernias Encountered } \\
\hline
\end{tabular}

\begin{tabular}{|c|c|}
\hline Type of Anaesthesia & Total \\
\hline Spinal anaesthesia/SA & 47 cases \\
\hline Epidural & 7 cases \\
\hline \multicolumn{2}{|c|}{ Duration of Operation (Minutes) } \\
\hline Mean & 38.6 minutes \\
\hline Range & $35-45$ minutes \\
\hline \multicolumn{2}{|c|}{ Table 2: Operative Results } \\
\hline
\end{tabular}

\begin{tabular}{|c|c|c|}
\hline \multicolumn{3}{|c|}{ Numeric Scale to describe Pain and Affect on ADLs. ${ }^{5}$} \\
\hline Description & Score & $\begin{array}{c}\text { Number of } \\
\text { Patients (\%) }\end{array}$ \\
\hline No pain & 0 & 0 \\
\hline $\begin{array}{l}\text { Mild pain or discomfort. No } \\
\text { interference with ADLs or } \\
\text { instrumental or advanced } \\
\text { ADLs }\end{array}$ & $1-2$ & 0 \\
\hline $\begin{array}{l}\text { Mild-to-moderate pain. More } \\
\text { distracting. Instrumental } \\
\text { ADLs may be impacted }\end{array}$ & 3 & $36(66.7)$ \\
\hline $\begin{array}{l}\text { Moderate pain; Limiting } \\
\text { activity (Instrumental and } \\
\text { advanced ADLs) }\end{array}$ & 4 & 0 \\
\hline $\begin{array}{c}\text { Moderate pain; Increasing } \\
\text { severity }\end{array}$ & $5-6$ & $16(29.6)$ \\
\hline $\begin{array}{l}\text { Moderate-to-severe pain } \\
\text { ADLs affected }\end{array}$ & 7 & $2(3.7)$ \\
\hline Severe & $8-9$ & 0 \\
\hline $\begin{array}{c}\text { Worse pain possible; } \\
\text { Immobilized or overwhelmed } \\
\text { by pain }\end{array}$ & 10 & 0 \\
\hline \multicolumn{3}{|c|}{ ADLs - Activities of Daily Living } \\
\hline \multicolumn{3}{|c|}{ Table 3: Pain Scoring Chart } \\
\hline
\end{tabular}

\section{CONCLUSION}

The results of this technique of adult hernia repair with continuous absorbable sutures of the external oblique aponeurosis appear promising. Since it does not use any foreign body, it has minimal complications with no recurrence of hernia or chronic groin pain. The continuous suturing saves time using only one packet of suture material. The procedure is simple and easy to perform and learn. It does not require complicated dissection or suturing nor does it use the weakened muscles or transversalis fascia for repair. In short adult inguinal hernia repair with continuous sutures of external oblique aponeurosis is safe and effective with favourable outcomes in terms of post-operative pain, return to work and recurrence and it can be the preferred choice for repair of adult inguinal hernia.

\section{REFERENCES}

1. Kingsnorth AN, Bennett DH, Hernias, et al. In: russel RCG, williams NS, bulstrode CJK, editors. Bailey \& love's short practice of surgery. London: arnold publishers, 2000;23rd ed:p 1143-62.

2. Sherman V, Maho JR, Brunicardi FC. Inguinal hernias. In Brunicardi FC, Andersen DK, Billiar TR, Dunn DL, Hunter JG, Mathews JB, et al. editors. Schwartz's principles of surgery. USA Mc Graw-Hill, 2010; $9^{\text {th }}$ ed:p 1305-42.

3. Desarda MP, Ghosh A. A comparative study of open mesh repair and desarda's no-mesh repair in a district hospital in India. East Centr Afr J Surg 2006;11(2):2834.

4. Owens WD, Felts JA, Spitnagel EL Jr. ASA physical status classification: a study of consistency of ratings. Anaesthesiology 1978;49(4):239-43.

5. Twaddle ML, Cooke KJ. Assesment of pain and common pain syndromes In: Von Roenn JH, Paice JA, Preodor ME, editors. Current diagnosis and pain management USA. Mc Graw- Hill 2006;1 ${ }^{\text {st }}$ ed:p 11-21.

6. Tobin GR, Clark S, Peacock EE Jr. A neuromuscular basis for development of indirect inguinal hernia. Arch Surg 1976;111(4):464-6.

7. Desarda MP. Surgical physiology of inguinal hernia repair- a study of 200 cases. BMC Surgery 2003;3(2):17.

8. Situma SM, Kaggwa S, Masiira NM, et al. Comparison of desarda versus modified bassini inguinal hernia repair: a randomized controlled trial. East Cent Afr J Surg 2009;14(2):70-6.

9. Rodriguez PL, Rodriguez FL, Rizo EP, et al. A new surgical approach in inguinal herniorrhaphy. Rev Cubana Cir 2004;43(2):25-8.

10. Lichtenstein IL, Shulman AG, Amid PK, et al. The tension-free hernioplasty. Am J Surg 1989;157(2):18893.

11. Desarda MP. No-mesh inguinal hernia repair with continuous absorbable sutures: a dream or reality? (a study of 229 patients). Saudi J Gastroenterol 2008;14(3):122-7.

12. Mitura K, Romanczuk M. Comparison between two methods of inguinal hernia surgery-lichtenstein and desarda. Pol Merk Lek 2008;24(143):392-5.

13. Mc Cormack K, Scott NW, Go PM, et al. Laparoscopic techniques versus open techniques for inguinal hernia repair. Cochrane Database Syst Rev 2003;(1):CD001785.

14. Bhattacharjee PK. Surgical options in inguinal hernia: which is the best? Ind J Surg 2006;68(4):191-200.

15. Porrero JL, Hidalgo $M$, Sanjuanbenito A, et al. The shouldice herniorrhaphy in the treatment of inguinal hernias: a prospective study on 775 patients. Hernia 2004;8(1):60-3.

16. Tanphiphat C, Tanprauyoon T, Sangsubhan C, et al. Laparoscopic versus open inguinal hernia repair: a randomized controlled trial. Surg Endosc 1998;12(6):846-51.

17. Shouldice EE. The treatment of the hernia. Ontario Med Rev 1953;1:1-14. 
18. Fleming WR, Elliott TB, Jones RM, et al. Randomized clinical trial comparing totally extraperitoneal inguinal hernia repair with the shouldice technique. Br J Surg 2001;88(9):1183-8.

19. Neumayer M, Giobbie HA, Jonasson O, et al. Open mesh versus laparoscopic mesh repair of inguinal hernia. $\mathrm{N}$ Engl J Med 2004;350(18):1819-27.

20. Nixon SJ, Jawaid H. Recurrence after inguinal hernia repair at ten years by open darn, open mesh and TEP-no advantage with mesh. Surgeon 2009;7(2):71-4.

21. Kurukahvecioglu O, Ege B, Yazicioglu O, et al. Polytetrafluoroethylene prosthesis migration into the bladder after laparoscopic hernia repair: a case report. Surg Laparosc Endosc Percutan Tech 2007;17(5):474-6.

22. Goswami R, Babor M, Ojo A. Mesh erosion into caecum following laparoscopic repair of inguinal hernia (TAPP): a case report and literature review. J Laparoendosc Adv Tech 2007;17(5):669-72.
23. Nicholson $\mathrm{S}$. Inguinal hernia repair. $\mathrm{Br} \quad \mathrm{J}$ Surg 1999;86(5):577-8.

24. Hay JM, Boudet JM, Fingerhut A, et al. Shouldice inguinal hernia repair in the male adult: the gold standard? a multicenter controlled trial in 1578 patients. Ann Surg 1995;222(6):719-27.

25. Kusnierczyk R, Lorek M. Inguinal hernia repair using own surgical technique-results of 500 operative procedures. Chir Pol 2003;5(3):145-53.

26. Desarda MP. New method of inguinal hernia repair:a new solution. ANZ J Surg 2001;71(4):241-4.

27. Khan N, Naeem M, Bangash A, et al. Early outcome of lichtenstein technique of tension-free open mesh repair for inguinal hernia. J Ayub Med Coll Abbottabad 2008;20(4):29-33. 